\title{
A Novel Splitting-Beam Laser Extensometer Technique for Kolsky Tension Bar Experiment
}

\author{
Xu Nie $\cdot$ Bo Song $\cdot$ Colin M. Loeffler
}

Received: 15 December 2014 / Accepted: 7 January 2015/Published online: 21 January 2015

(C) Society for Experimental Mechanics, Inc 2015

\begin{abstract}
A modified laser occlusive extensometer technique was developed to measure the specimen strain with reasonably high and tunable resolutions in Kolsky tension bar experiments. This new technique used a novel concept where a laser beam was split into two independent sections to track the displacement histories of the incident and transmission bar ends, respectively, with independent resolutions. This technique ensures highly precise small strain measurements without sacrificing the capacity for large strain measurement. The uncertainty caused by rigid body motion of the specimen during dynamic strain measurement, which is induced by slight variation of laser intensity along the gage length, was also minimized in this technique. The validation test on Vascomax ${ }^{\circledR}$ maraging C250 alloy demonstrated that the new technique was capable of making both small and large strain measurements in Kolsky tension bar experiments, which is also applicable to Kolsky compression bar experiments.
\end{abstract}

Keywords Kolsky tension bar - Laser extensometer . Small strain measurement $\cdot$ High strain rate

\section{Introduction}

Kolsky bars, also known as split Hopkinson bars, have been extensively employed to characterize the dynamic

X. Nie $(\varangle) \cdot$ C. M. Loeffler

Mechanical and Energy Engineering, University of North Texas, Denton, TX 76207, USA

e-mail: xu.nie@unt.edu

B. Song

Sandia National Laboratories, Albuquerque, NM 87185, USA stress-strain response of materials [1]. In Kolsky bar experiments, the strain measurement in the specimen is more challenging than stress measurement, particularly when in need of investigating the stress-strain response of materials at small strains. It has been a common practice in Kolsky bar experiments to use the reflected pulse to calculate the specimen strain [1]. However, in many cases, especially in Kolsky tension bar experiments, the reflected pulse may not be reliable due to complex interfacial conditions between the tensile specimen and the bars [2]. Therefore, non-contact optical techniques for the specimen strain measurement have been proposed and developed [3, $4]$ in lieu of the conventional specimen strain calculation with the reflected pulse.

Laser "extensometer" has become a straight forward method of non-contact measurement of specimen strain in Kolsky bar experiments. In this technique, a uniform laser sheet is generated to cover the entire gage section of the specimen. When the specimen is deformed, the change in the gage length of the specimen results in the change in the pass-through light intensity collected by a high-frequencyresponse laser detector located behind the specimen [3, 4]. Through proper calibration, the temporal history of the gage length of the specimen being deformed can be measured to calculate the corresponding specimen strain history. Satisfactory results were reported by $\mathrm{Li}$ et al. [5] on using this technique to measure the dynamic tensile behavior of several metallic alloys and composites with a Kolsky tension bar system. Recently, this method has even been implemented to a small-diameter Kolsky tension bar system for single fiber testing [6, 7]. However, both the incident and transmission bar ends in Kolsky bar experiments move in the same direction but at different velocities. A rigid body motion may be superimposed to the absolute deformation of the specimen. If there is a slight variation in laser intensity 
along the measurement gage section (which is often the case in experimental practice), the rigid body motion will induce a small output signal obscuring the actual specimen deformation, particularly at small strains. In order to demonstrate the variation in the laser intensity, the local laser sensitivities were carefully calibrated over a small range $(\sim 200 \mu \mathrm{m})$ at three adjacent locations with approximately $300 \mu \mathrm{m}$ apart. In each calibration, a high-resolution differential translation stage was applied to generate a $10-\mu \mathrm{m}$ increment of gap size. Figure 1 summarizes the calibration results in terms of the gap size versus laser detector voltage output. The relationships between the two parameters were observed to be linear at all three locations but with slightly different slopes due to the variation in local laser intensity. In addition to the noise introduced by the rigid body motion, the measurement resolutions in current laser extensometer techniques are limited particularly at small strains due to the relatively long gage section in Kolsky tension bar experiments. Using a laser system to track the displacement at the incident-bar/specimen interface has been recently proposed to improve the resolution [8]. However, the $100-\mu \mathrm{m}$ resolution of the laser detector used in [8] was still not sufficient for obtaining reliable elastic stress-strain response of the material under investigation.

Digital image correlation (DIC) is another non-contact technique that has been improved and extended to Kolsky bar experiments for dynamic full-field strain measurements in the specimen [9-13]. However, the resolution of the high-rate DIC highly depends on the frame rate, maximum number of frames, and image resolution of the camera, in addition to a number of other challenges including reliable patterning and proper lighting on the specimen surface. The high-rate DIC provides highly valuable information on the

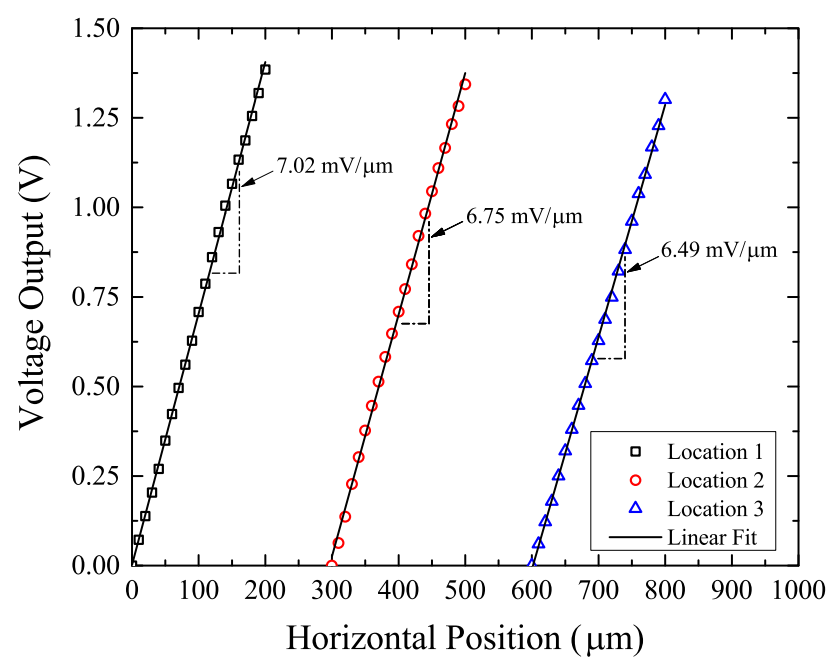

Fig. 1 Calibration of laser sensitivity at different locations within the gauge length. The variation of sensitivity indicates slight change in laser intensity uniformity of deformation in the specimen during dynamic loading but insufficient data points and/or image resolution to construct a precise stress-strain curve due to the extreme difficulty in acquiring large quantity of high-resolution images at very high frame rates [8].

In this study, we split a single laser beam into two sections to independently track the motions of the incident and transmission bar ends. The motions of the incident and transmission bar ends were measured with independent and tunable resolutions, giving more opportunities to improve the resolution of resultant stress-strain curve. As an example, the new splitting-beam laser technique was employed to dynamically characterize the tensile stressstrain response of a VascoMax ${ }^{\circledR}$ maraging C250 alloy with a Kolsky tension bar.

\section{Experiments}

The general concept of the splitting-beam technique is shown in Fig. 2. A $50 \mathrm{~mW}$ line laser and optical lens system were applied to a Kolsky bar setup to generate a collimated sheet laser scanning through the gap between the incident and transmission bars. The pass-through laser beam was then divided into two independent sections using a right-angle prism mirror. The apex of the right angle serves as a virtual reference line diverting the laser into two opposite directions, with each portion exclusively tracking the motion of either the incident or transmission bar end. This design prevented the possible interference between the two laser channels and made the displacement measurements at the incident-bar/specimen and specimen/ transmission-bar interfaces independent. Each individual signal was then collected with a laser detector (Thorlabs PDA 36A). The Thorlabs laser detector has a tunable resolution with a correlated frequency response. At $100 \mathrm{kHz}$ or higher bandwidth that is usually required for Kolsky bar experiments [2], the laser detector is capable of measuring the displacement with a resolution of approximately $100 \mathrm{~nm}$, which corresponds to a strain resolution of $0.0016 \%$ for a $6.35-\mathrm{mm}$-long specimen. The utilization of two independent laser detection systems allows setting the resolutions and bandwidths for displacement measurement at the incident-bar/specimen and specimen/transmissionbar interfaces and independently. In Kolsky bar experiments, the transmission bar moves at a lower velocity than the incident bar. Therefore, the detector responsible for the measurement at the transmission bar side needs to be set with a higher resolution due to the smaller full-scale displacement output. Such a characteristic improves the accuracy for small-strain measurement without sacrificing the superiority of traditional laser extensometers on largestrain measurement. 
Fig. 2 A 3-D model schematic of the splitting-beam laser extensometer technique for Kolsky bars. Note that the laser beam is diverted at the apex of the right-angle prism mirror, which creates a virtual reference line for independent displacement tracking

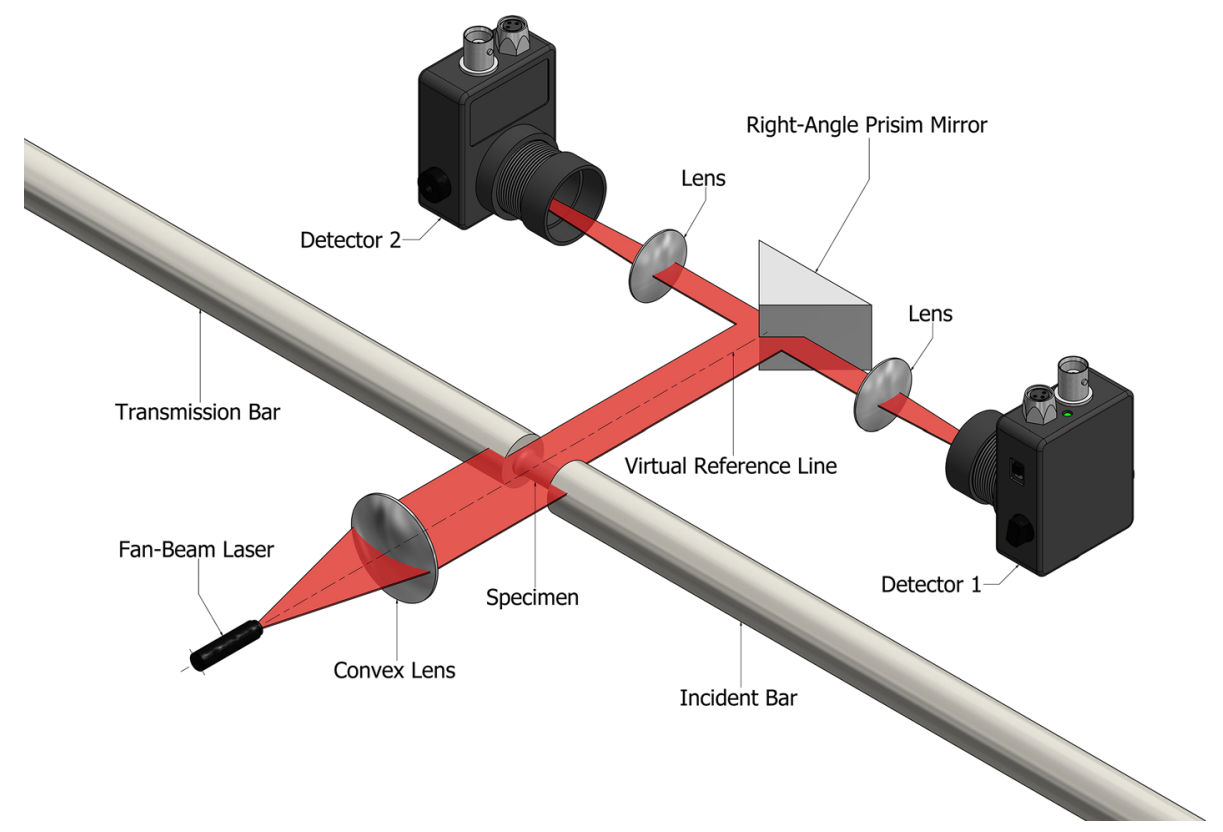

The new dynamic splitting-beam laser extensometer was applied to Kolsky tension bar experiments on an ATI Vascomax $^{\circledR}$ maraging C250 alloy. The raw material was normalized at $927^{\circ} \mathrm{C}$ for $1 \mathrm{~h}$ followed by water quenching and then annealed at $816^{\circ} \mathrm{C}$ for $1 \mathrm{~h}$ before rapid air cooling. After machining, the tensile specimens were tempered at $482{ }^{\circ} \mathrm{C}$ for $3 \mathrm{~h}$ and then air cooled. The specimens for Kolsky tension bar experiments were made into dog-bone-shaped cylinders with threads at both ends. The tensile specimens had a gage section of $3.18 \mathrm{~mm}$ in diameter and $6.35 \mathrm{~mm}$ in length, which transitioned into $1 / 2^{\prime \prime}-20$ threaded ends. The Kolsky tension bar used here has been described previously in [8, 14]. The 19.05-mm diameter incident and transmission bars that were made of Vascomax ${ }^{\circledR}$ maraging C350 alloy were 3,658- and 2,134$\mathrm{mm}$ long, respectively. Both incident and transmission bars had the same $\left(1 / 2^{\prime \prime}-20\right)$ female threads at the specimen ends such that the tensile specimen can be directly threaded into the bar ends without the need of adapters. The experimental detail is referred to Ref. [15].

A set of original oscilloscope records for the tensile test is shown in Fig. 3. It is noted that a strain gage was directly attached on the specimen surface to compare with the measurements from the laser system at small specimen strains up to $2 \%$. As shown in Fig. 3, the specimen strain gage failed shortly after the specimen was dynamically loaded. It also needs to be pointed out that the specimen strain gage exactly recorded the specimen deformation within the area covered by the strain gage, while the splitting-beam laser extensometer measured the total displacements of both gage and transitional (non-gage) sections. Additional corrections are required to obtain the actual deformation over the specimen gage section only $[8,15]$. In this study, the same correction procedure described in Ref. $[8,15]$ has been followed to calculate the actual specimen strain in the gage section from the measurement results of the splitting-beam laser extensometer. The stress-strain curve based on the laser extensometer measurements with proper correction was thus obtained and compared with that based on the direct specimen strain gage measurement, the results of which are shown in Fig. 4. Again, the stress-strain curve based on the direct specimen strain gage measurement is valid only when the strain is smaller than $2 \%$. Figure 4 clearly shows that the splitting-beam laser technique measured a very close stress-strain behavior in the elastic region as the specimen strain gage did. When the specimen deformation passed the peak tensile stress of 2.5 $\mathrm{GPa}$, the two techniques started to show significant deviation partly because the specimen strain gage reached its limit at the strain of $2 \%$. Another possible reason for this deviation is that the correction of the plastic strain in the gage section from the laser extensometer measurements was erroneous because the correction was based on the assumption of perfect plasticity [8], which is obviously not the case for the $\operatorname{Vascomax}^{\circledR}$ maraging C250 alloy investigated in this study. Based on the tensile stress-strain curve shown in Fig. 4, the Vascomax ${ }^{\circledR}$ maraging C250 alloy may exhibit very early localized plastic deformation, such as necking, which resulted in erroneous calculation of the specimen plastic strain with the laser extensometer technique. Regardless, the accuracy of the new splitting-beam laser technique on capturing dynamic small-strain deformation is clearly demonstrated in addition to its capability of recording the complete specimen deformation history. 
Fig. 3 Original oscilloscope record of the Kolsky tension bar validation test

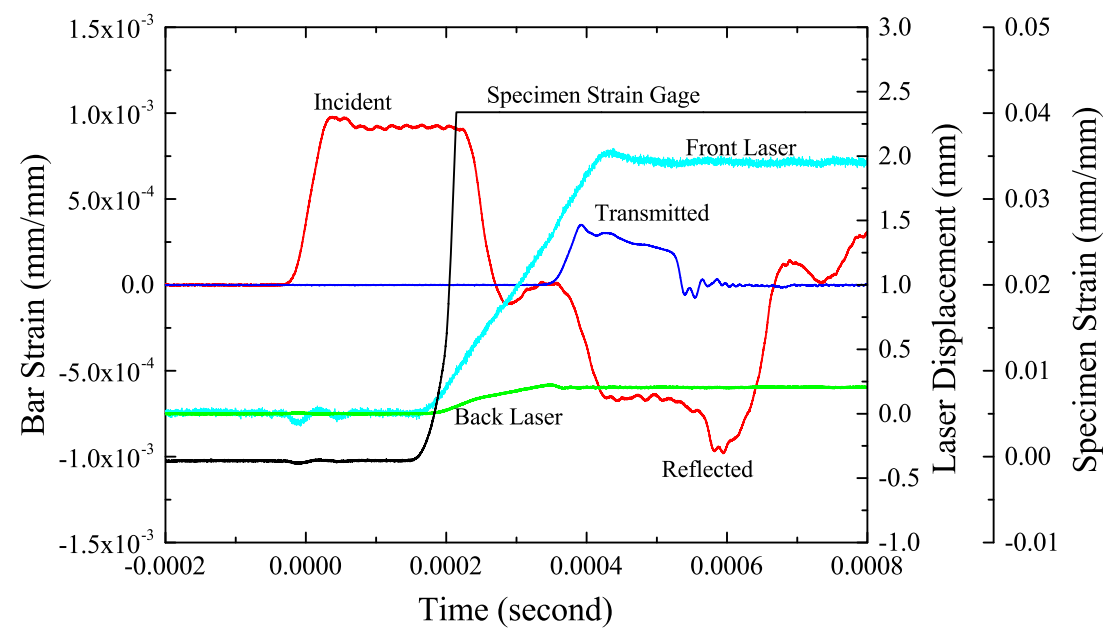

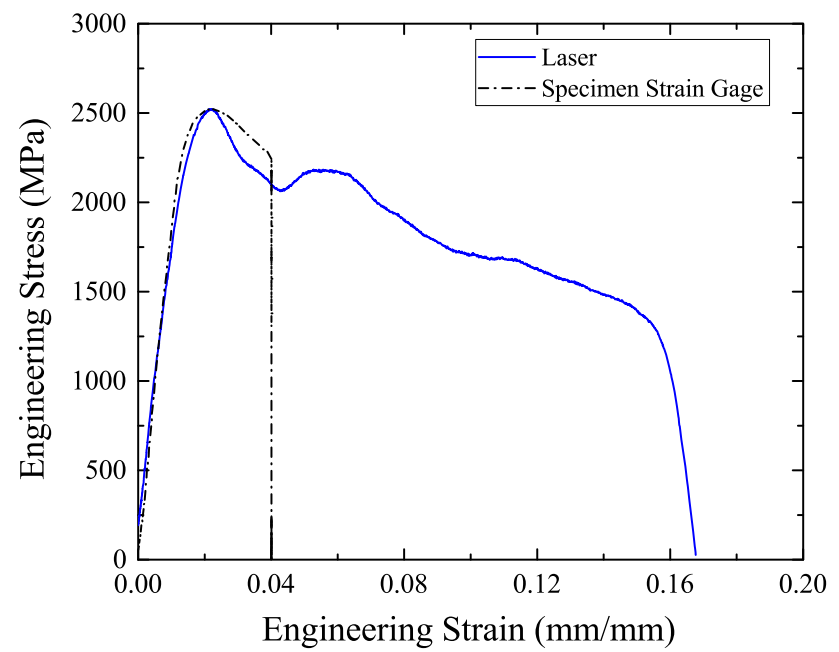

Fig. 4 Tensile stress-strain curves obtained from different strain measurement techniques

\section{Conclusion}

Through adopting the innovative idea of splitting a single laser beam, the conventional laser occlusive extensometer was modified for Kolsky bar experiments to simultaneously measure the small- and large-strain deformation of the specimen with high resolutions and bandwidths. This innovative design also allows for independently tuned resolution of each photo detector, which improves the accuracy of specimen strain measurement, particularly at small strains. The validation test was carried out with a Vascomax ${ }^{\circledR}$ maraging C250 alloy on a Kolsky tension bar. The results showed that the elastic portion of the dynamic tensile stress-strain curve measured with the splittingbeam laser extensometer technique agreed well with the direct specimen strain gage measurement, demonstrating its capabilities for both small and large specimen deformation measurements.
Acknowledgments This work was sponsored by the United States Department of Energy (DOE) Office of Space and Defense Power Systems (NE-75). The authors gratefully acknowledge the support and guidance of Ryan D. Bechtel of the US Department of Energy. The authors also acknowledge the discussion with and support of Peter E. Wakeland and Michael D. Furnish for the dynamic tensile experiments in this study. Sandia National Laboratories is a multiprogram laboratory managed and operated by Sandia Corporation, a wholly owned subsidiary of Lockheed Martin Corporation, for the US Department of Energy's National Nuclear Security Administration under contract DE-AC04-94AL85000.

\section{References}

1. Kolsky H (1949) An investigation of the mechanical properties of materials at very high rates of loading. Proc Phys Soc B 62:676-700

2. Chen W, Song B (2011) Split Hopkinson (Kolsky) bar: design, testing and applications. Springer, Berlin

3. Ramesh KT, Narasimhan S (1996) Finite deformations and the dynamic measurement of radial strains in compression Kolsky bar experiments. Int J Solids Struct 33(25):3723-3728

4. Ostwaldt D, Klepaczko JR, Klimanek P (1997) Compression tests of polycrystalline $\alpha$-iron up to high strains over a large range of strain rates. J Phys IV France 7(C3):385-390

5. Li Y, Ramesh K (2007) An optical technique for measurement of material properties in the tension Kolsky bar. Int J Impact Eng 34:784-798

6. Lim J, Chen W, Zheng Q (2010) Dynamic small strain measurements of Kevlar 129 single fibers with a miniaturized tension Kolsky bar. Polym Test 29:701-705

7. Hudspeth M, Nie X, Chen W, Lewis R (2012) Effect of loading rate on mechanical properties and fracture morphology of spider silk. Biomacromolecules 13:2240-2246

8. Song B, Antoun BR, Jin H (2013) Dynamic tensile characterization of a 4330-V steel with Kolsky bar techniques. Exp Mech 53:1519-1529

9. Barthelat F, Wu Z, Prorok BC, Espinosa HD (2003) Dynamic torsion testing of nanocrystalline coatings using high-speed photography and digital image correlation. Exp Mech 43:331-340

10. Mates SP, Rhorer R, Everett RK, Simmonds KE, Bagchi A (2009) High strain rate tissue simulant measurement using digital image correlation. In: Proceedings of the SEM annual conference, Albuquerque NM, June 1-4 
11. Siviour CR, Grantham SG (2009) High resolution optical measurements of specimen deformation in the split Hopkinson pressure bar. Imaging Sci J 57:333-343

12. Dunand M, Gary G, Mohr D (2013) Load-inversion device for the high strain rate tensile testing of sheet materials with Hopkinson pressure bars. Exp Mech 53:1177-1188

13. Gilat A, Schmidt TE, Walker AL (2009) Full field strain measurement in compression and tensile split Hopkinson bar experiments. Exp Mech 49:291-302
14. Song B, Antoun BR, Connelly K, Korellis J, Lu W-Y (2011) Improved Kolsky tension bar for high-rate tensile characterization of materials. Meas Sci Technol 22:045704

15. Song B, Wakeland PE, Furnish MD (submitted) Dynamic tensile characterization of Vascomax ${ }^{\circledR}$ maraging C-250 and C-300 alloys. J Dynamic Behavior Mater 